\title{
Diacronie
}

Studi di Storia Contemporanea

$N^{\circ} 10,2$ | 2012

Digital History: la storia nell'era dell'accesso

\section{Au-delà de la gestion des références bibliographiques: Zotero}

\section{Frédéric Clavert}

\section{(2) OpenEdition}

\section{Journals}

\section{Édition électronique}

URL : http://journals.openedition.org/diacronie/2851

DOI : 10.4000/diacronie.2851

ISSN : 2038-0925

Éditeur

Association culturelle Diacronie

Référence électronique

Frédéric Clavert, «Au-delà de la gestion des références bibliographiques: Zotero », Diacronie [En ligne], $N^{\circ} 10,2$ | 2012, document 6, mis en ligne le 29 juin 2012, consulté le 19 avril 2019. URL : http:// journals.openedition.org/diacronie/2851; DOI : 10.4000/diacronie.2851 


\title{
Diacronie
}

6/

\section{Au-delà de la gestion des références bibliographiques: Zotero}

\author{
Frédéric CLAVERT *
}

Né au sein du Centre d'Histoire et des Nouveaux Médias de l'Université de George Mason en Virginie, Zotero se distingue comme étant un logiciel capable de s'adapter au nouveau environnement numérique dans lequel travaille l'historien ajourd'hui. Ses multiples fonctionnalités - passées en revue dans ces pages - s'averent être des outils incotournables pour la gestion et l'exploitation des différents corpus de soures. Ces données, issues désormais de tout type de supports, pourront enfin être structurées et organisées aux fins d'une publication, ainsi que réutilisées pour le travail collaboratif et la mise en ligne de bibliographies.

\section{Introduction}

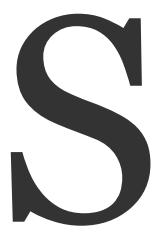
elon les mots du Roy Rosenzweig Center for History and New Media (CHNM), Zotero est «[...] un outil libre, pratique pour vous aider à collecter, organiser, citer et partager les sources de vos recherches. Il fonctionne exactement là où vous travailler - au sein du navigateur web lui-même» ${ }^{1}$. Le CHNM a été créé par l'historien spécialiste de Public History Roy Rosenzweig dans les années $1990^{2}$. Parti de presque rien, ce centre est aujourd'hui un acteur majeur de l'histoire et des sciences humaines numériques³.

\footnotetext{
${ }^{1}$ Zotero [zoh-TAIR-oh] is a free, easy-to-use tool to help you collect, organize, cite, and share your research sources. It lives right where you do your work-in the web browser itself». 'Zotero | Home', URL: < https://www.zotero.org/ > [consulté 21 mai 2012].

2 Pour avoir un aperçu des travaux de Roy Rosenzweig (décédé en 2007): ROSENZWEIG, Roy, Clio wired: the future of the past in the digital age, New York, Columbia University Press, 2011.
} 
Piloté par Dan Cohen 4 , directeur du CHNM, et par Sean Takats 5 , directeur des projets de recherche du CHNM, le projet Zotero fait suite à un précédent logiciel, Scribe, qui n'est plus aujourd'hui développé6. Si Scribe était très orienté vers la prise de note - et reste supérieur sur ce point - Zotero a été conçu sur le principe selon lequel l'environnement (numérique) du chercheur - historien ou non - a évolué et est désormais centré sur le navigateur web. Ce changement de paradigme, qui reflète une réelle évolution des méthodologies et du traitement des sources de l'histoire, fait de Zotero un programme polyvalent, à même de gérer de nombreux aspects de la recherche historienne.

Zotero est devenu, au fil de ses versions, plus qu'un logiciel de gestion des références bibliographiques. Il peut, si vous le souhaitez, devenir un outil central pour l'organisation et la gestion de votre recherche. Il vous aidera à respecter des bonnes pratiques informatiques sans même que vous ne vous en rendiez compte. Ces «bonnes pratiques» seront nécessaires, notamment, pour pouvoir travailler sur les informations que vous avez stockées dans votre bibliothèque Zotero. Pour montrer en quoi zotero est un outil plutôt simple d'utilisation et touchant à de très nombreux aspects de votre recherche, cet article s'attachera à brièvement expliquer pourquoi choisir Zotero en premier lieu, à effectuer une revue des principales fonctionnalités de ce logiciel en second lieu et, en dernier lieu, à montrer comment Zotero peut être utilisé pour exploiter votre corpus de sources.

\section{Pourquoi Zotero?}

Lorsqu'un historien se rend dans un centre d'archives, lorsqu'il prend des notes en lisant une publication scientifique, lorsqu'il utilise le web, lorsqu'il écrit, il produit des données. Comment gérer ces dernières? C'est à cette question, finalement, que Zotero répond. Il permet la collecte des données issues de tout type de supports, tout en

\footnotetext{
3 Pour avoir une meilleur idée des acteurs des Digital Humanities, il est possible de se référer à deux cartographies des centres de recherches de cette discipline. La première a été élaborée par centerNet, réseau international des centre d'Humanités numériques:

URL: < http://digitalhumanities.org/centernet/centers/ >; et celle du PIREH (Université Paris I - Panthéon Sorbonne), plus francophone:

URL < http://pireh.univ-paris1.fr/DHfrancophone/ > [consultés le 18 juin 2012].

4 Voir son blog, qui traite de nombreux aspects des Digital Humanities: Dan Cohen's Digital Humanities Blog, URL:< http://www.dancohen.org/ > [consulté le 18 juin 2012].

5 Le blog de Sean Takats comprend des billets sur zotero, mais également sur d'autres sujets liés au Digital Humanities: The Quintessence of Ham, URL $:<$ http://quintessenceofham.org/ > [consulté le 18 juin].

6 «Center for History and New Media-Scribe», URL: < http://chnm.gmu.edu/tools/scribe/ >, [consulté le 21 avril 2012].
} 
insistant plus particulièrement sur le navigateur web. Il les stocke sur votre ordinateur et les synchronise éventuellement en ligne. Zotero n'est pas un service web, mais bien une application - qui existe sous deux formes: celle d'une extension pour le navigateur Firefox ou celle d'un programme indépendant.

Sorti en 2007, aujourd'hui à sa version 3 , Zotero est classé parmi les logiciels de gestion de données bibliographiques. Il fait face au géant Endnote ${ }^{7}$ ou Mendeley ${ }^{8}$, développé par une société commerciale londonienne.

Zotero se distingue de ses concurrent principaux car il est fondé sur des principes compatibles avec l'éthique universitaire: libre et financé de manière non ambiguë par des bourses, notamment des fondations Andrew W. Mellon et Alfred P. Sloan', et par l'achat, non obligatoire, d'espace disque pour la synchronisation facultative des fichiers sur les serveurs de Zotero. Les directeurs du projet Zotero sont des universitaires qui exercent une activité d'enseignement et de recherche et qui sont, sans aucun doute, ses premiers et plus exigeants utilisateurs. Son développement est soutenu par un centre de recherche et de ressources appartenant à l'Université George Mason (Virginie), de très bon niveau. Le résultat est un logiciel qui respecte votre travail et peut s'intégrer dans de multiples traditions épistémologiques. En outre, Zotero vous permet de le quitter, par un export facile de vos données - ainsi restez-vous maître de ces dernières, d'autant plus qu'il est «libre»:

L'expression «logiciel libre» veut dire que le logiciel respecte la liberté de l'utilisateur et de la communauté. En gros, les utilisateurs ont la liberté d'exécuter, de copier, de distribuer, d'étudier, de modifier et d'améliorer le logiciel. Avec ces libertés, les utilisateurs (à la fois individuellement et collectivement) contrôlent le programme et ce qu'il fait pour eux. ${ }^{10}$

\footnotetext{
7 En 2008-2009, l'éditeur d'EndNote, Thomson-Reuters, a tenté une procédure en justice contre Zotero - ou plutôt contre l'Université George Mason -, sans succès. Il était reproché aux créateurs de Zotero d'avoir utilisé des techniques contraires à la licence d'EndNote pour permettre à Zotero d'être compatible avec le logiciel de Thomson-Reuters. TAKATS, Sean, «Thomson Reuters Lawsuit Dismissed», in The Quintessence of Ham,

URL: < http://quintessenceofham.org/2009/06/04/thomson-reuters-lawsuit-dismissed/ > [consulté le 21 avril 2012].

8 «Free reference manager and PDF organizer | Mendeley»,

URL: < http://www.mendeley.com/ > [consulté le 21 juin 2012].

9 COHEN, Dan, «Building a Sustainable Zotero Project», in Zotero,

URL: < http://www.zotero.org/blog/building-a-sustainable-zotero-project/ > [consulté le 8 juin 2012].

10 FREE SOFTWARE FOUNDATION, «Qu'est-ce qu'un logiciel libre?»,

URL: < http://www.gnu.org/philosophy/free-sw.html >, [consulté le 1 juin 2012].
} 
Peu d'historiens peuvent analyser le code source de Zotero ${ }^{11}$. Toutefois, la logique est communautaire: parmi ses utilisateurs, certains sont capables de contrôler et de contribuer à son développement. Des éléments clés pour les chercheurs sont ainsi garantis. En premier lieu, le fait que leurs données ne seront pas trafiquées par le programme qu'ils utilisent ${ }^{12}$.

Cette dimension collective a également un autre intérêt: un logiciel libre disposant d'une communauté énergique d'usagers se doit d'être à l'écoute des besoins de ces derniers. Le dynamisme des forums de Zotero $^{13}$ et des sites fournissant une aide ou une réflexion sur son usage ${ }^{14}$ permet à un utilisateur de trouver plus facilement une solution à un problème auquel il serait confronté. Au-delà des problèmes techniques, il est également possible de contribuer au code de Zotero, soit pour résoudre des dysfonctionnements, soit pour ajouter des fonctionnalités ou encore pour créer des logiciels le complétant.

Enfin, Zotero respecte des protocoles et langages ouverts connus. Lorsqu'il importe une citation directement depuis votre navigateur, il peut utiliser les spécifications Dublin Core ${ }^{15}$, ce qui le rend compatible avec de nombreux sites. De même, les styles de citation de Zotero respectent le CSL ${ }^{16}$ qui est utilisé par d'autres logiciels concurrents.

Pour toutes ces raisons, Zotero est un logiciel adapté aux besoins de la recherche en histoire. Nous allons, dans la partie suivante, tenter de montrer de manière plus concrète en quoi ses fonctionnalités appuient l'historien dans ses recherches.

\footnotetext{
${ }^{11}$ Le code source est «un ensemble d'instructions écrites dans un langage de programmation informatique de haut niveau, compréhensible par un être humain entraîné, permettant d'obtenir un programme pour un ordinateur». WIKIPEDIA CONTRIBUTORS, «Code source», in Wikipédia, URL : < http://en.wikipedia.org/ > [consulté le 21 juin 2012]. 2012.

${ }_{12}$ Ce souci d'utiliser un logiciel libre correspond à ce qui a été affirmé par l'article $10 \mathrm{du}$ Manifeste des Digital humanities écrit collectivement à Paris en 2010. «Manifeste des Digital humanities | ThatCamp Paris 2010», URL: < http://tcp.hypotheses.org/318 > [consulté le 15 février 2012].

13 «Zotero Forums», URL: < http://forums.zotero.org/ > [consulté le 21 juin 2012].

14 Pour les Francophones: le blog Zotero francophone (URL: <http://zotero.hypotheses.org/>) maintenu notamment par l'auteur de cet article et La Boîte à outils des historiens (URL: <http://www.boiteaoutils.info/>) [consultés le 21 juin 2012]

${ }^{15}$ La Dublin Core Metadata Initiative définit un standard de métadonnées. Les métadonnées sont des données servant à définir ou à décrire d'autres données. «DCMI Home: Dublin Core ${ }^{\circledR}$ Metadata Initiative (DCMI)», URL: < http://dublincore.org/ > [consulté le 21 juin 2012].

${ }^{16}$ Le Citation Style Language (CSL) est un langage permettant de définir des styles de citation. «CitationStyles.org | The Citation Style Language - open and free citation styles»,

URL: < http://citationstyles.org/ > [consulté 21 juin 2012].
} 


\section{Principales fonctiones de Zotero}

Nous divisons, un peu artificiellement, les fonctionnalités de Zotero en trois parties: fonctions élémentaires, fonctions en ligne, fonctions avancées. Mais avant tout, nous nous pencherons sur son installation.

\subsection{Installation}

Pour installer Zotero, vous devrez vous rendre sur le site officiel, http://www.zotero.org, ou vous trouverez un bouton Download qui vous mènera vers une page vous décrivant la marche à suivre en fonction:

- de la version de Zotero que vous souhaitez (version Standalone si vous n'utilisez pas ou peu Firefox ou si vous préférez utiliser plusieurs navigateurs / version Firefox si ce dernier est votre navigateur principal) ${ }^{17}$;

- de votre système d'opération (Mac OS X, Windows ou Linux);

- de votre traitement de texte (MS Word ou Libre/OpenOffice) pour le plug-in permettant d'utiliser votre base Zotero directement dans votre traitement de texte.

17 HEIMBURGER, Franziska, «La Boite à Outils des Historiens: Zotero - version Firefox ou Stand-alone?», in La Boîte à outils des historiens, URL: < http://www.boiteaoutils.info/2012/o5/zotero-version-firefox-ou-stand-alone.html > [consulté le 19 juin 2012]. 


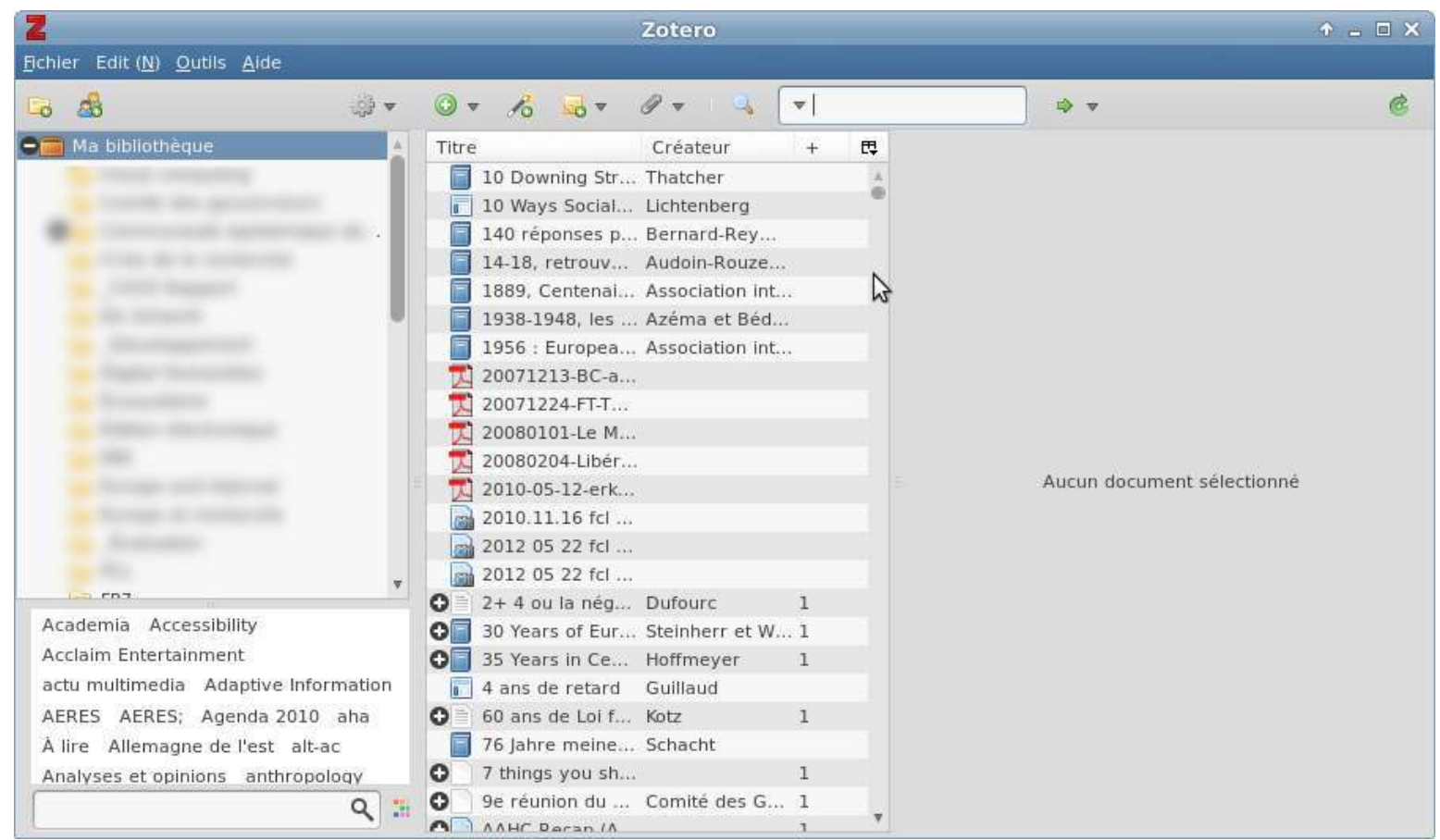

Illustration 1: Zotero Standalone (application séparée)

Si vous avez installé la version dite «Standalone», vous devrez également installer les «connecteurs» qui permettent de créer un lien entre les navigateurs comme Chrome ou Safari et Zotero. Ils sont aussi disponibles sur la page de téléchargement de Zotero. 


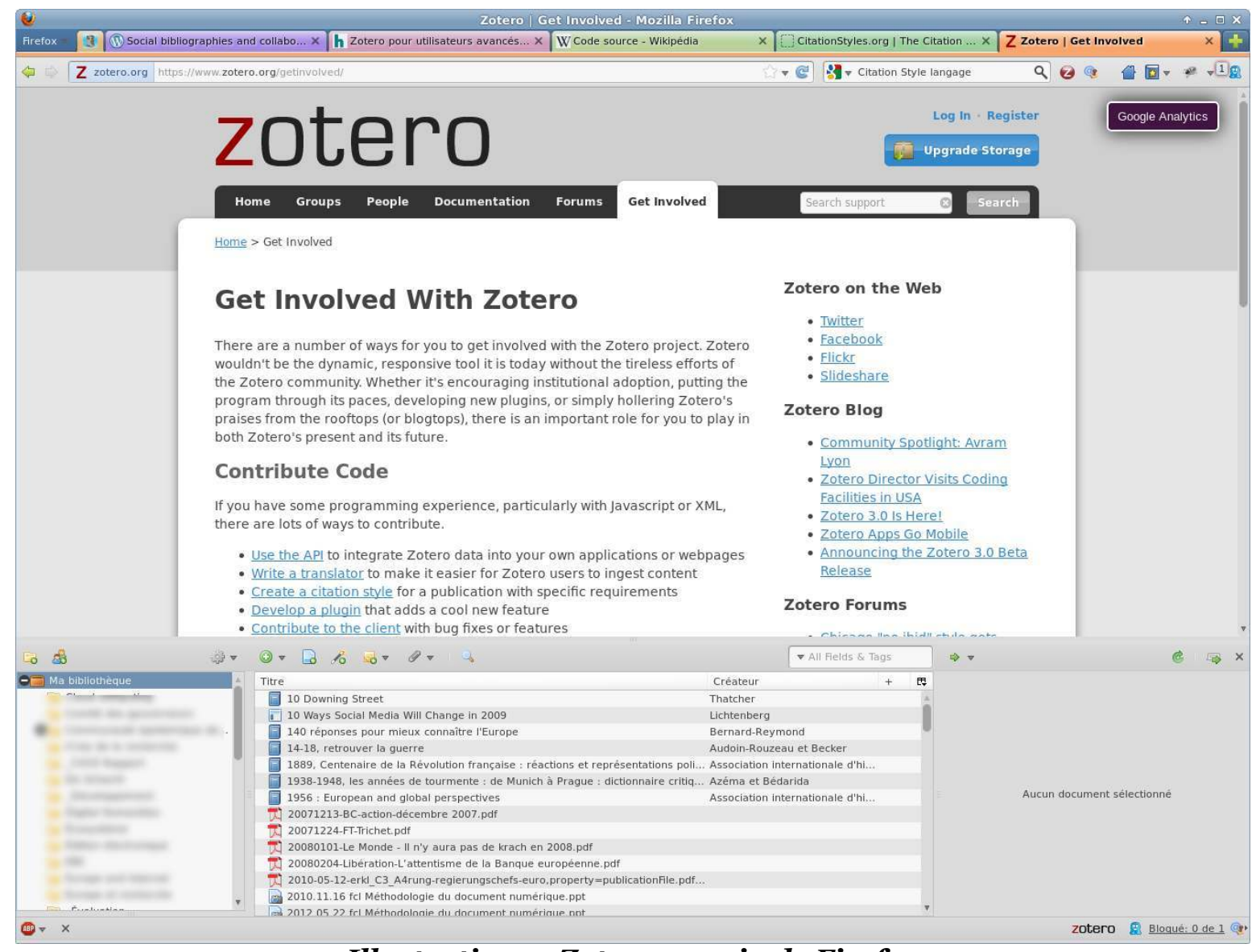

\section{Illustration 2: Zotero au sein de Firefox}

Une fois Zotero installé, soit vous cliquez sur l'icône de l'application (version Standalone), soit vous cliquez sur l'icône Zotero en bas à droite de votre fenêtre Firefox (version Firefox). Zotero s'affiche alors (Illustrations $1 \& 2$ ).

Maintenant que vous avez installé Zotero, regardons ses fonctionnalités élémentaires et, notamment, la collecte des références bibliographiques et l'utilisation des références stockées dans zotero directement à partir du traitement de texte. 


\subsection{Fonctions élémentaires'8}

La première et plus essentielle fonctionnalité de Zotero est de collecter les références bibliographiques. Il y a deux moyens pour cela: par saisie manuelle ou par saisie automatique. Dans le second cas, il suffit de cliquer sur une icône qui est dans la barre d'adresse de votre navigateur (Illustration 3). Cette icône change en fonction de la nature de la référence bibliographique (livre, article, billet de blogs, etc). Les types d'objet que Zotero reconnaît sont heureusement nombreux.

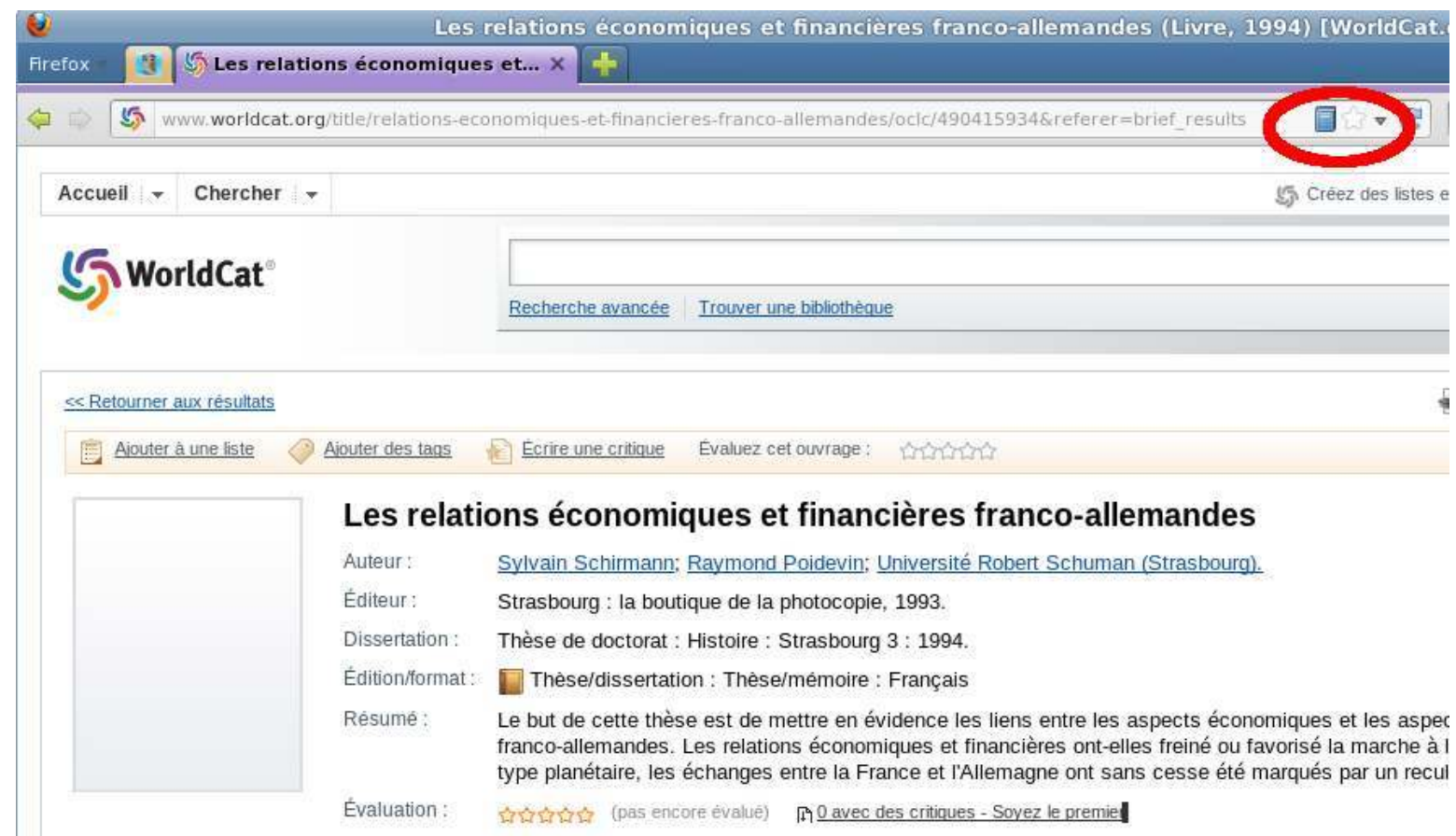

Illustration 3: Importer une référence bibliographique de la barre d'adresse du navigateur

En utilisant un site web comme WorldCat ${ }^{19}$, méta-catalogue qui réunit les collections d'un très grand nombre de bibliothèques publiques dans le monde, il deviendra très rare que vous soyez obligés d'entrer manuellement une référence bibliographique. Dans la plupart des cas, les sites web des bibliothèques universitaires et/ou nationales sont compatibles.

${ }^{18}$ Nous décrivons ici les usages de Zotero. Pour une explication de son utilisation pas-à-pas, nous renvoyons à l'excellente présentation de Franziska Heimburger et Émilien Ruiz: RUIZ, Émilien, «La Boite à Outils des Historiens: Introduction à Zotero 3.0 : nouveau tutoriel!», in $L a$ Boîte à outils des historiens,

URL: < http://www.boiteaoutils.info/2012/o6/introdution-zotero-30-nouveau-tutoriel.html > [consulté 1 juin 2012].

19 «WorldCat.org», URL: < http://www.worldcat.org/ > [consulté le 21 juin 2012]. 
Pour les articles scientifiques, des bases de données telles que JSTOR, cairn, revues.org, persee.fr - et bien d'autres - fonctionnent avec Zotero. En outre, lorsque vous importez automatiquement une référence bibliographique, Zotero peut y adjoindre une capture d'écran. Vous pouvez vous-même, lorsque vous téléchargez l'article sous forme électronique - en PDF par exemple -, l'associer à la référence bibliographique correspondante: Zotero gère également vos fichiers. Vous pourrez ajouter des notes à vos références bibliographiques et les stocker dans votre bibliothèque Zotero.

Il y a deux autres moyens automatiques de récupérer une référence bibliographique dans Zotero: en indiquant un DOI ou un $\mathrm{ISBN}^{20}$ ou en récupérant les méta-données d'un PDF. Cette dernière fonctionnalité, par expérience, ne fonctionne pas toujours bien, car le PDF doit être connu de Google Scholar, le service de recherche sur les publications scientifiques de Google.

La saisie manuelle se fait simplement, en remplissant des champs libellés de manière explicite qui apparaitront dans le panneau de droite.

Vous pourrez associer des mots-clés à vos références bibliographiques, pour faciliter une recherche. Vous pouvez indiquer des éléments «connexes» - c'est-à-dire associer deux ou plusieurs références bibliographiques les unes aux autres. Cette fonctionnalité permet de lier, par exemple, un article aux autres publications scientifiques qu'il cite et ainsi «formaliser» une sorte de réseau de citations.

Le panneau central de Zotero affiche l'ensemble des références stockées dans la base de données. Le panneau de gauche affiche les «collections» qui vous permettent de classer ces références - par thème, par centres d'archives, par dates... en fonction de vos envies et de votre manière de travailler.

La seconde fonctionnalité primordiale de Zotero est la possibilité de pouvoir citer et formater automatiquement des références bibliographiques stockées dans votre bibliothèque Zotero directement pendant l'écriture d'un article ou d'un livre. Des plugins, faciles à installer, existent pour MS Word et pour Apache OpenOffice / LibreOffice / NeoOffice. Ils sont compatibles avec Windows, Mac OS X et Linux. Ces plug-ins rajoutent une barre d'outils dans votre traitement de texte afin de vous permettre de citer et de formater les citations et d'insérer une bibliographie dans un document. Il

20 DOI signifie «Digital Object Identifier». Il s'agit d'identifiants uniques attribués à des ressources numériques. L'ISBN est numéro international standardisé du livre qui permet d'identifier de manière unique un livre. 
existe des centaines de styles de citation adaptés à de nombreuses revues ou éditeurs scientifiques $^{21}$.

\subsection{Fonctions en ligne}

Zotero est utilisable comme une application totalement hors connexion. Toutefois, vous pourrez également bénéficier des services qui sont liés au site http://www.zotero.org.

La fonctionnalité en ligne majeure est la synchronisation de vos données Zotero sur plusieurs ordinateurs - et, grâce à ZotPad ${ }^{22}$ et Zandy ${ }^{23}$, avec certaines tablettes ou téléphones portables. Il y a deux types de synchronisation possibles : les références bibliographiques elles-mêmes d'une part et les fichiers que vous avez éventuellement attachés à ces références d'autre part. Cette distinction s'explique par le fait que les fichiers prennent nettement plus de place que les références bibliographiques. Ainsi, au-delà de $100 \mathrm{Mb}$ de fichiers, vous devrez, pour les synchroniser, soit acheter de l'espace sur le site de Zotero.org, soit disposer d'un accès à un compte WebDAV ${ }^{24}$ souvent fourni par les universités ${ }^{25}$.

Depuis quelques mois, Zotero.org a facilité l'accès et la modification en ligne de votre bibliothèque. Vous pourrez accéder à l'ensemble de vos données Zotero (y compris les fichiers, si vous les synchronisez avec Zotero.org) à partir de n'importe quel navigateur web - donc également à partir d'ordinateurs qui ne vous appartiennent pas - et rajouter des références bibliographiques, des notes, des collections... mais pas de fichier pour le moment.

\footnotetext{
${ }^{21}$ Vous les trouverez à cette adresse: URL: < http://www.zotero.org/styles > [consulté le 21 juin 2012]. Pour les styles francophones, vous pouvez vous rendre, si vous ne trouvez pas de styles correspondant à vos besoins, sur le site «CSL francophone» vous pouvez faire référence à, URL: $<$ https://trello.com/board/csl-france/4e8f4ee92adc2aoooo9616d3 > [consulté le 21 juin 2012].

${ }^{22}$ ZotPad. An iPad client for Zotero,

URL: < http://www.zotpad.com/ > [consulté le 21 juin 2012].

${ }^{23}$ LYON, Avram, «Zandy User Guide», in Contact in the Russian Empires,

URL : < http://www.gimranov.com/avram/w/zandy-user-guide > [consulté le 21 juin 2012].

${ }^{24}$ Le WebDAV est un protocole vous permettant de manipuler dossiers et fichiers distants facilement.

25 Rappelons que la synchronisation de vos données sur plusieurs appareils n'est pas une sauvegarde. Synchroniser ses données signifie qu'en cas d'erreur de manipulation, celle-ci se reportera sur l'ensemble de vos appareils. Pour plus d'informations sur des méthodes simples de sauvegarde, voir: HEIMBURGER, Franziska, «Les bonnes résolutions... ou comment mettre en place un système complet et automatique de sauvegarde», in La Boîte à outils des historiens, URL: < http://www.boiteaoutils.info/2010/o1/les-bonnes-resolutions-ou-comment.html >, [consulté le 6 juin 2012].
} 
Zotero.org offre également la possibilité de créer des groupes, privés, publics avec adhésion fermée, publics avec adhésion ouverte. Dans le premier cas, seuls les membres peuvent voir le contenu du groupe et l'adhésion au groupe ne peut se faire que sur invitation de l'administrateur du groupe - souvent son créateur. Il permet de partager non seulement les références bibliographiques, mais également les fichiers. Dans le second cas, les références bibliographiques seront publiques, les fichiers ne pourront être partagés (pour des raisons de droits d'auteur), mais l'adhésion au groupe se fera après modération par l'administrateur. Dans le troisième cas, les références bibliographiques seront partagées et toute personne intéressée par le groupe pourra en devenir membre et y contribuer sans modération.

Les groupes apparaîtrons dans votre application Zotero, dans le panneau de gauche, en-dessous de votre bibliothèque. Vous pourrez contribuer au travail du groupe à partir de Zotero par simple glissé-déposé ou en créant les références directement dans le groupe. Nous verrons dans la partie suivante, que le travail fait dans ces groupes peut être reporté sur un site web, soit par utilisation directe de l'API ${ }^{26}$ de Zotero.org, soit par l'usage d'un système de gestion de contenu ${ }^{27}$ adapté.

Zotero.org propose d'autres fonctionnalités: suivre le travail de quelqu'un et être suivi par les autres ${ }^{28}$; un système de messagerie propre à Zotero; une page vous permettant d'avoir un CV en ligne, avec possibilité de faire appel à vos propres publications stockées dans Zotero, ce qui permet d'avoir une liste de publication à jour sans y penser.

\subsection{Fonctions avancées ${ }^{29}$}

Nous aborderons ici quelques fonctions avancées de Zotero: les cartes, les chronologies et l'API.

\footnotetext{
26 WIKIPEDIA CONTRIBUTORS, "Interface de programmation», in Wikipédia, URL : < http://en.wikipedia.org/ > [consulté le 21 juin 2012].

27 WIKIPEDIA CONTRIBUTORS, «Système de gestion de contenu», in Wikipédia, URL : < http://en.wikipedia.org/ > [consulté le 21 juin 2012].

${ }^{28}$ Ces fonctionnalités «sociales» sont toutefois peu développées.

29 Cette section est inspirée d'un billet du blog Zotero francophone: CLAVERT, Frédéric, «Zotero pour utilisateurs avancés», in Zotero francophone,

URL: < http://zotero.hypotheses.org/268 > [consulté le 21 juin 2012].
} 


\subsubsection{Cartes}

En installant Zotero Maps, un plug-in spécifique ${ }^{30}$, il est possible de représenter une bibliothèque Zotero sur une carte, qui peut représenter divers champs, dont, naturellement, le lieu de publication.

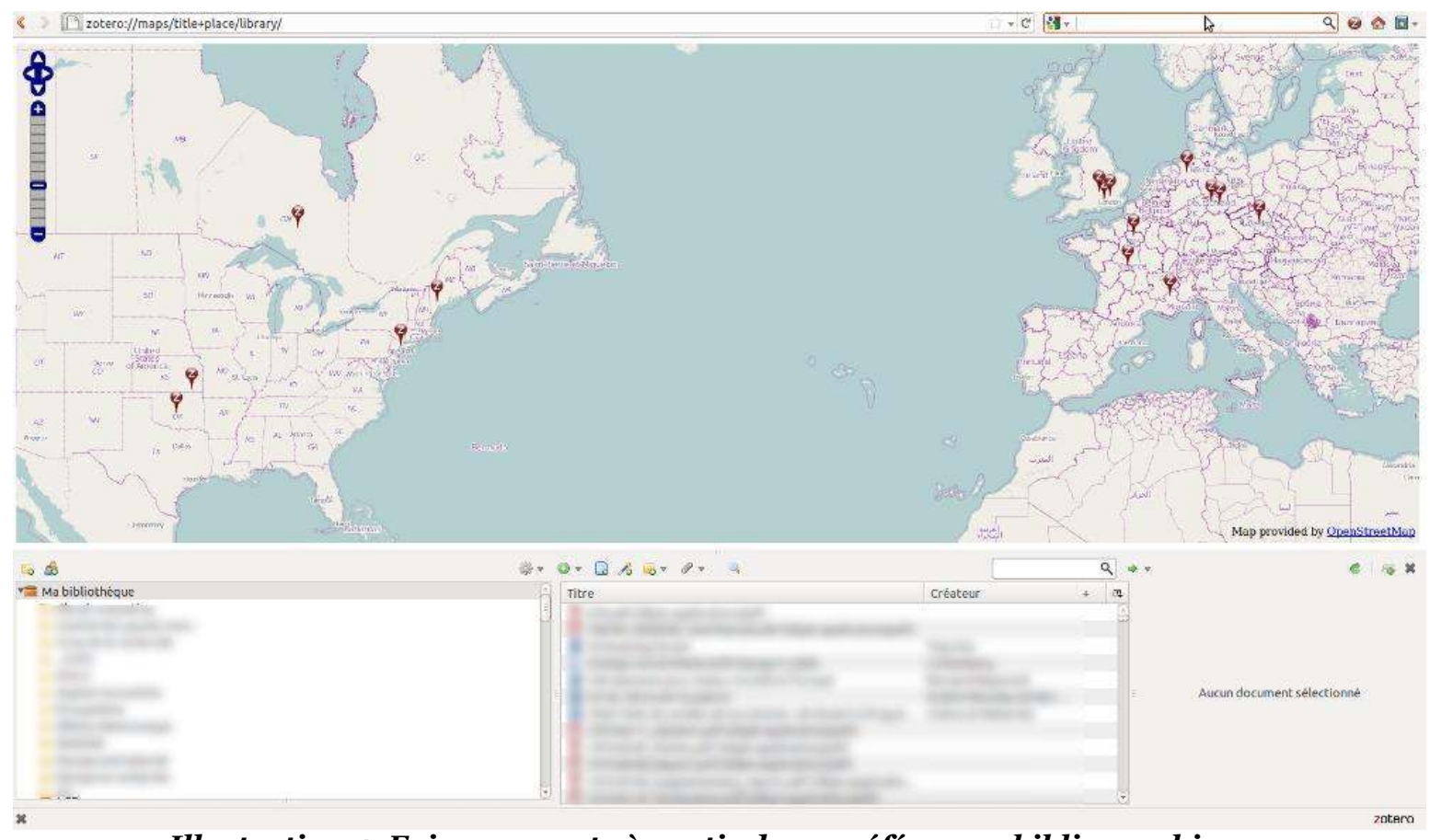

Illustration 4: Faire une carte à partir de vos références bibliographiques

Ainsi, peut-on visualiser, par exemple, la répartition géographique des sources utilisées pour un travail de recherche. Imaginons-nous quelques instants à faire de l'histoire de l'intégration européenne. Si vos sources sont essentiellement liées au Parlement européen, elles peuvent avoir, notamment, trois origines géographiques: Luxembourg, Strasbourg et Bruxelles. Une représentation géographique montrera ce que pèsent les deux premières par rapport à la troisième dans le processus de décision parlementaire européen.

${ }^{30}$ Ce plug-in est normalement fait pour Firefox. Toutefois, la version Zotero standalone est capable d'utiliser ces plug-ins, via le menu «Outils» et le sous-menu «Extension». Pour une description détaillée et l'adresse du plug-in, voir OWENS, Trevor, «Zotero Maps: Visualize Your Zotero Library on the Globe», in Zotero, URL: < http://www.zotero.org/blog/zotero-mapsvisualize-your-zotero-library-on-the-globe/ >, [ consulté le 6 juin 2012]. La version la plus récente de ce plug-in est téléchargeable à l'adresse, URL: < https://github.com/zotero/zoteromaps/downloads $>$ [consulté le 21 juin 2012]. 


\subsubsection{Chronologies}

Zotero Maps pourra être complété par la création de chronologies à partir de vos références bibliographiques stockées dans Zotero. Contrairement à Zotero Maps, l'outil de représentation chronologique est intégré à Zotero - aucune installation supplémentaire n'est nécessaire. Zotero utilise un ensemble de logiciels libres appelés Simile et développé par le medialab du MIT ${ }^{31}$. La représentation chronologique obtenue est présentée en trois grandes parties: jours, mois et années. Vous pouvez surligner jusqu'à quatre mots clés sur votre représentation chronologique.

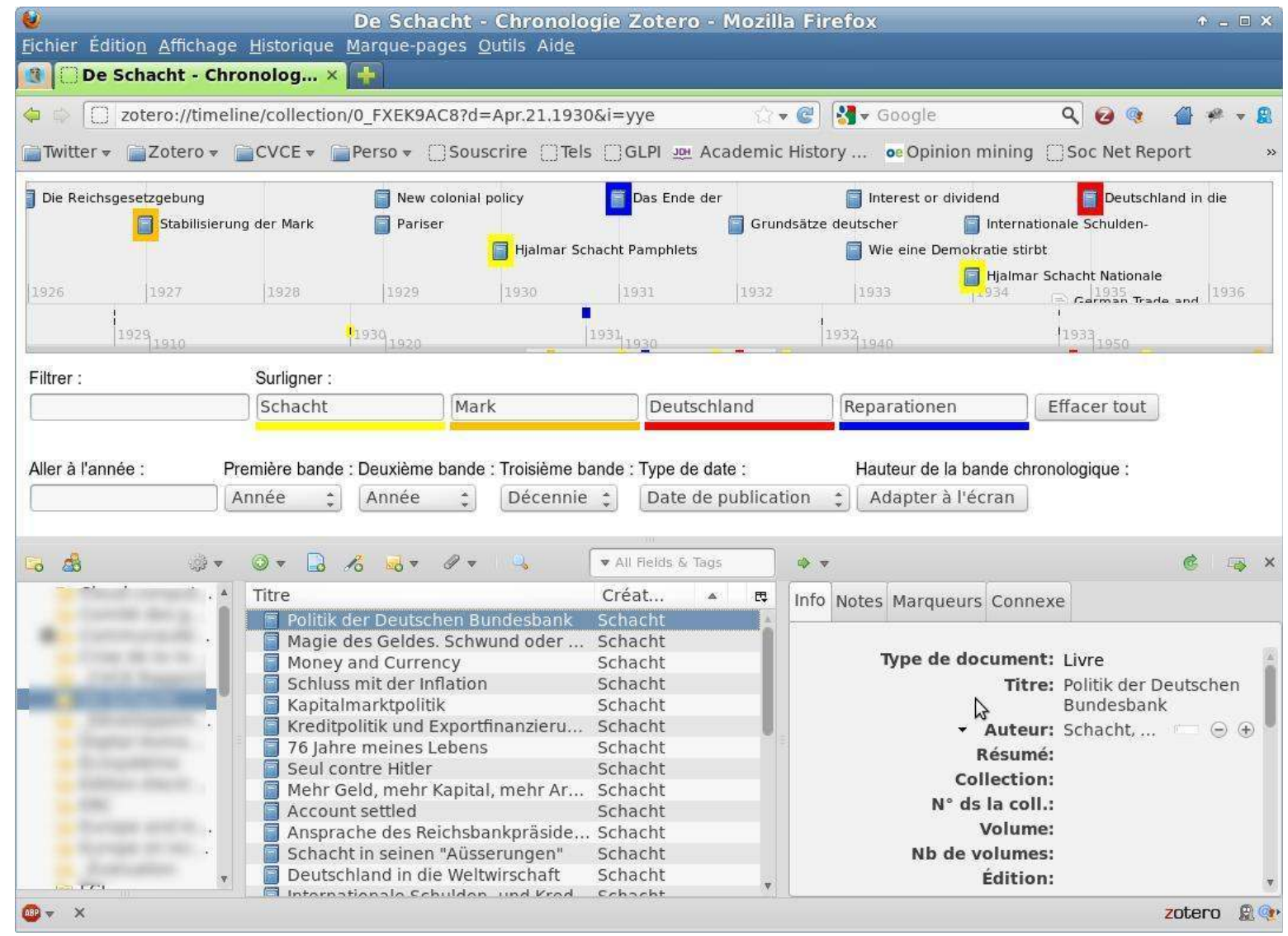

Illustration 5: Une chronologie des publications de Hjalmar Schacht, président de la Reichsbank

En visualisant, par exemple, une collection où vous stockez les principales publications de votre champ de recherche, vous pourrez graphiquement voir quels sont les principales étapes de la littérature scientifique que vous avez consultée, les évolutions d'un débat historiographique, etc.

${ }^{31}$ Semantic Interoperability of Metadata and Information in unLike Environments, URL : < http://simile.mit.edu/ > [consulté le 21 juin 2012]. 
D'autres plug-ins rajoutent des fonctionnalités à Zotero. Ainsi, Zotfile ${ }^{32}$ vous permettra-t-il de mieux gérer les fichiers attachés aux références bibliographiques, mais, surtout, d'envoyer vos documents au format PDF sur votre tablette ou téléphone portable, de les lire et les y annoter avec les logiciels ad hoc, puis de les rapatrier sur votre ordinateur. Vous pourrez alors extraire les annotations que vous avez effectuées sur votre terminal mobile et les transformer en notes Zotero.

Malheureusement, ces greffons ont un cycle de vie parfois raccourci: certains n'ont été développés que pour une version précise de Zotero et deviennent obsolètes lorsque celui-ci est mis-à-jour. Cela a été notamment le cas de Vertov, qui apportait des fonctionnalités d'annotations des vidéos et qui n'est désormais plus utilisable33.

\subsubsection{API}

Une API est une interface de programmation - c'est-à-dire, pour faire bref et caricatural, la manière dont des programmes, y compris des sites web, communiquent entre eux et partagent des fonctionnalités. Dans le cas de Zotero.org, l'API permet à des programmes tiers - les excellents Zandy pour Android ou ZotPad pour iOS par exemple - de manipuler votre bibliothèque Zotero. C'est aussi une manière d'utiliser vos références bibliographiques dans un site web. Cela s'applique à vos propres collections, mais également aux références bibliographiques collectées dans un groupe Zotero ${ }^{34}$.

Pour utiliser l'API, il faut être un utilisateur enregistré de Zotero.org et se connecter pour obtenir une clé, que l'on peut demander dans son profil (settings > Feeds/API). Ceci est un point qui peut paraître très technique, mais qui vous servira si vous souhaitez utiliser vos références bibliographiques sur un site web.

Ainsi, le plug-in Zotpress pour Wordpress ${ }^{35}$ permet d'intégrer vos données Zotero à votre blog, site ou carnet de recherche ${ }^{36}$. L'une des utilisation possibles est de faire figurer sur un site une bibliographie élaborée collectivement via un groupe sur

32 «ZotFile - Zotero Addon to manage your attachments»,

URL: < http://www.columbia.edu/ jpl2136/zotfile.html > [consulté le 21 juin 2012].

33 «Vertov», URL: < http://digitalhistory.concordia.ca/vertov/ > [consulté le 21 juin 2012].

34 Vous pourrez trouver une documentation sur l'API de Zotero à cette adresse: «Dev:server_api [Zotero Documentation]»,

URL: < http://www.zotero.org/support/dev/server_api > [consulté le 21 juin 2012].

35 «WordPress > Blog Tool, Publishing Platform, and CMS», URL: < http://wordpress.org/ > [consulté le 21 juin 2012].

36 «WordPress > Zotpress « WordPress Plugins»,

URL: < http://wordpress.org/extend/plugins/zotpress/> [consulté le 21 juin 2012]. Une extension similaire existe pour SPIP. Voir CLAVERT, Frédéric, «Utiliser zotero avec le CMS SPIP», in Zotero francophone, URL: < http://zotero.hypotheses.org/453 > [consulté le 6 juin 2012]. 
Zotero.org. À ce titre, un exemple particulièrement intéressant est la bibliographie collaborative mise en place par l'International Society for First World War Studies ${ }^{37}$. Ce travail collectif a l'avantage de faire appel à des historiens ayant de la Première Guerre mondiale, ayant des connaissances différentes, pouvant ensemble constituer une bibliographie très complète. Les compétences comme le temps sont ainsi partagés.

Une utilisation plus simple de l'API est d'utiliser les flux RSS engendrés par Zotero.org. Un flux RSS est un fichier écrit automatiquement qui permet de s'informer des mises à jours d'un site web38. Zotero.org en produit pour votre bibliothèque lorsque vous la synchronisez, mais également pour chacune de vos collections. La plupart des systèmes de gestion des contenus disposent d'extensions qui reprendront le flux RSS, le formateront, et le reporteront sur votre site web, la plupart du temps dans un encadré. Ainsi, le site web de l'Association française d'Histoire économique utilise-t-il le flux RSS d'un groupe Zotero pour reporter les dix dernières publications de ses membres 39 .

Les fonctions avancées de Zotero permettent bien d'autres développements qu'il serait trop long de développer ici. Dans la section suivante, nous allons tenter de montrer comment exploiter plus avant le corpus de sources que peut devenir votre bibliothèque Zotero.

\section{Aller plus loin: Zotero pour exploiter un corpus de sources}

Le travail de l'historien repose, notamment, sur la collecte et l'exploitation de sources primaires et secondaires, action dont Zotero facilite l'exécution. Pour la méthodologie concrète, nous vous renvoyons à un billet de Franziska Heimburger sur le site La boite à outils des historiens ${ }^{40}$. Elle y aborde à la fois l'utilisation de Zotero, mais également celle des appareils photographiques en centre d'archives.

Une fois vos sources collectées et stockées dans Zotero, il est possible de considérer votre bibliothèque Zotero - ou certaines de ses collections - comme un corpus à part

37 Son fonctionnement est expliqué (en anglais) dans HEIMBURGER, Franziska, «New collaborative bibliography of First World War Studies», in International Society for First World War Studies, URL: < http://www.firstworldwarstudies.org/?p=973 > [consulté le 6 juin 2012]. $3^{8}$ WIKIPEDIA CONTRIBUTORS, «RSS», in Wikipédia, URL : < http://en.wikipedia.org/ > [consulté le 21 juin 2012].

39 Encadré «Lectures et publications», dans la colonne de droite du site,

URL: < http://afhe.hypotheses.org/ > [consulté le 21 juin 2012].

40 HEIMBURGER, Franziska, «La photographie numérique des sources - conseils, astuces, méthodes», in La Boite à Outils des Historiens,

URL: < http://www.boiteaoutils.info/2011/o8/la-photographie-numerique-des-sources.html > [consulté le 6 juin 2012]. 
entière. Ce corpus peut, par exemple, être connecté à un outil comme Omeka ${ }^{41}$. Ce dernier est un logiciel du CHNM qui permet notamment de collecter et d'exposer des sources sur le web. Cette connexion permettra de publier votre corpus en ligne - bien entendu dans le respect du droit d'auteur. C'est ce qu'a fait en 2011 Radu Suciu, postdoctorant à l'Université Paris Diderot (Paris 7), pour le site Medical Case Studies on Renaissance Melancholy: Online Publication Project ${ }^{42}$.

Plus avancé encore est l'utilisation que le CHNM fait de Zotero pour collecter des sources actuelles. L’idée date de 2001. Le CHNM a mis en ligne un site ${ }^{43}$ permettant la collecte en direct de témoignages - sous de nombreuses formes: poèmes, dessins, photographies... - sur les attentats du 11 septembre. Sur cette base, il est possible de savoir ce qu'on fait les citoyens états-uniens le 11 septembre 2001 et, par exemple, de savoir quelle chaîne de télévision ils ont regardé. On peut alors comprendre où la très influente Fox News est née : dans les régions rurales 44.

En 2001, Zotero n'existe pas encore. Mais cette première expérience explique probablement pourquoi Zotero a été prévu, dès sa conception, pour stocker des sources avant de les publier en ligne. Récemment, le CHNM a utilisé une combinaison de Zotero et d'Omeka pour entretenir la mémoire du mouvement de réaction à la crise financière «Occupy»45. Une précieuse source pour les sciences humaines et sociales.

Pour les plus téméraires, le rapport Data mining with criminal intent ${ }^{46}$ montre comment utiliser Zotero - parmi d'autres outils - pour stocker des sources collectées en ligne et, ensuite, travailler sur ces données avec des outils très avancés comme Mathematica 47 . Plus concret, The Programming historian ${ }^{48}$ vous apprendra à

${ }^{41}$ URL: < http://www.omeka.net/ > [consulté le 21 juin 2012].

${ }^{42}$ SUCIU, Radu, «Medical Case Studies on Renaissance Melancholy», in Medical Case studies on Renaissance Melancholy-Online publication project,

URL: < http://www.melancholystories.com/ > [consulté le 6 juin 2012]. Les principes qui ont guidé cette publication ont été exposés à la conférence Digital Humanities 2011 à Stanford: SUCIU, Radu, «Medical Case Studies on Renaissance Melancholy: Online Publication Project», in Digital Humanities 2011, URL:

< http://dh2011abstracts.stanford.edu/xtf/view?docId=tei/ab-217.xml;query=;brand=default > [consulté le 6 juin 2012].

43 The september 11 digital archive. Saving the histories of september 11, 2001,

URL : < http://911digitalarchive.org/ > [consulté le 16 juin 2012].

44 COHEN, Dan, «Mapping What Americans Did on September 11», URL:

< http://www.dancohen.org/blog/posts/mapping_what_americans_did_on_september_11 > [consulté le 17 septembre 2012].

45 ROY ROSENZWEIG CENTER FOR HISTORY AND NEW MEDIA, «Occupy Archive», \#Occupy Archive, URL: < http://occupyarchive.org/ > [consulté le 6 juin 2012].

${ }^{46}$ COHEN, Dan, et al., «Data Mining with Criminal Intent», 2011.

47 WIKIPEDIA CONTRIBUTORS, «Mathematica», in Wikipédia, URL : < http://en.wikipedia.org/ > [consulté le 21 juin 2012].

48 TURKEL, William J., MACEACHERN, Alan, «The Programming historian», 
développer un script pour collecter automatiquement des sources en ligne et les stocker dans votre bibliothèque Zotero.

URL: < http://niche.uwo.ca/programming-historian/index.php/Main_Page > [consulté le 15 septembre 2008]. Une seconde version est en préparation. 


\section{Conclusion}

Zotero est un outil très complet, qui peut accompagner le chercheur, qu'il soit historien ou non, dans toutes les étapes de sa recherche, de la collecte de ses sources à la publication, qu'elle soit sur papier ou en ligne. Bien qu'une forte fonctionnalité des programmes comme Zotero soit la gestion des références bibliographiques et des citations lors de la rédaction d'un article, nous espérons avoir attiré votre attention sur les autres usages, plus avancés, de Zotero, qui, tous, permettent de travailler sur vos données. Ainsi pourrez vous découvrir des éléments utiles pour votre recherche qu'il aurait été difficile, voire impossible, de faire ressortir sans l'utilisation de ce logiciel.

Pour le simple utilisateur, Zotero permet facilement et de façon transparente de gérer et structurer ses sources. Pour l'utilisateur plus avancé, il permettra de réutiliser ces données pour le travail collaboratif et la mise en ligne de bibliographies. Pour les plus téméraires, il sera un outil de fouille de données (data mining) et de récolte de sources en ligne.

Zotero est finalement un outil puissant, d'un usage plutôt simple, qui contribue à la pénétration des usages numériques dans la recherche historienne, qui, petit-à-petit, voit ses méthodes transformées 49 .

49 Retrouvez la bibliographie de cet article sur le site web de l'auteur :

URL: < http://www.clavert.net/ > [consulté le 21 juin 2012]. 


\section{* L'auteur}

Frédéric Clavert a étudié les sciences politiques et l'histoire de l'intégration européenne à l'Université de Strasbourg et à l'Université de Leeds. Après sa thèse sur «Hjalmar Schacht, financier et diplomate» soutenue à l'Université de Strasbourg en décembre 2006, ses recherches s'orientent aujourd'hui vers les relations entre les banquiers centraux et la construction européenne. Il a enseigné l'histoire des relations internationales, l'histoire de la construction européenne et les sciences politiques de l'Europe à l'Institut des Hautes Études Européennes et à l'Institut d'Études Politiques de l'Université de Strasbourg. Il est maintenant chercheur au Centre Virtuel de la Connaissance sur l'Europe. Il y développe notamment une réflexion méthodologique sur la pratique de l'histoire contemporaine à l'ère numérique. Ses récents publications incluent: Contemporary history in the digital age, PIE Peter Lang (2011) et Hjalmar Schacht, financier et diplomate: 1930-1950, Bruxelles, PIE - Peter Lang (2009).

URL: < http://studistorici.com/progett/autori/\#Clavert >

\section{Per citare questo articolo:}

CLAVERT, Frédéric, «Au-delà de la gestion des références bibliographiques: Zotero», Diacronie. Studi di Storia Contemporanea: Digital History: la storia nell'era dell'accesso, 29/6/2012,

URL:<http://www.studistorici.com/2012/06/29/clavert_numero_10/ >

\section{Diacronie Studi di Storia Contemporanea $\beta$ www.diacronie.it}

Risorsa digitale indipendente a carattere storiografico. Uscita trimestrale. redazione.diacronie@hotmail.it

Comitato di redazione: Marco Abram - Giampaolo Amodei - Jacopo Bassi - Luca Bufarale - Alessandro Cattunar - Alice De Rensis Barbara Galimberti - Deborah Paci - Fausto Pietrancosta - Matteo Tomasoni - Luca Zuccolo

Diritti: gli articoli di Diacronie. Studi di Storia Contemporanea sono pubblicati sotto licenza Creative Commons 2.5. Possono essere riprodotti a patto di non modificarne i contenuti e di non usarli per fini commerciali. La citazione di estratti è comunque sempre autorizzata, nei limiti previsti dalla legge. 